\title{
Development of a Dynamic Operational Scheduling Algorithm for an Independent Micro-Grid with Renewable Energy "
}

\author{
Shin'ya OBARA ${ }^{* *}$ \\ **Tomakomai National College of Technology, \\ Nishikioka 443, Tomakomai, HOKKAIDO 0591275, Japan \\ E-mail: obara@indigo.plala.or.jp
}

\begin{abstract}
A micro-grid with the capacity for sustainable energy is expected to be a distributed energy system that exhibits quite a small environmental impact. In an independent micro-grid, "green energy," which is typically thought of as unstable, can be utilized effectively by introducing a battery. In the past study, the production-of-electricity prediction algorithm (PAS) of the solar cell was developed. In PAS, a layered neural network is made to learn based on past weather data and the operation plan of the compound system of a solar cell and other energy systems was examined using this prediction algorithm. In this paper, a dynamic operational scheduling algorithm is developed using a neural network (PAS) and a genetic algorithm (GA) to provide predictions for solar cell power output. We also do a case study analysis in which we use this algorithm to plan the operation of a system that connects nine houses in Sapporo to a micro-grid composed of power equipment and a polycrystalline silicon solar cell. In this work, the relationship between the accuracy of output prediction of the solar cell and the operation plan of the micro-grid was clarified. Moreover, we found that operating the micro-grid according to the plan derived with PAS was far superior, in terms of equipment hours of operation, to that using past average weather data.
\end{abstract}

Key words: Micro-Grid, Operation Planning, Energy Storage, Renewable Energy, Neural Network, Weather Prediction

\section{Introduction}

Micro-Grid technology with the capacity for sustainable energy operation has been widely discussed recently from the point of view reducing the environmental impact of society ${ }^{1)-3}$. In these setups, the operation optimization program installed in the controller of a combined system is the most important aspect of the technology for determining the performance of the system ${ }^{4}$. However, because an output prediction for the green energy contribution to the system is required, the dynamic operation plan of a system that combines conventional energy equipment (for example, a diesel engine, a gas engine, a fuel cell, etc.) and green-energy equipment can be very difficult to design. In this work, we use a neural network (NN) to obtain output predictions for a solar cell. Weather data from the past 14 years (amount of solar radiation and outside temperature) is fed into the learning process of the NN. This NN production-of-electricity prediction algorithm was developed by the author and is described to be PAS in ${ }^{5)}$.

Power fluctuations are known to occur in systems that utilize green energy on an independent micro-grid and that experience large or rapid changes in load ${ }^{6}$. Given this, 


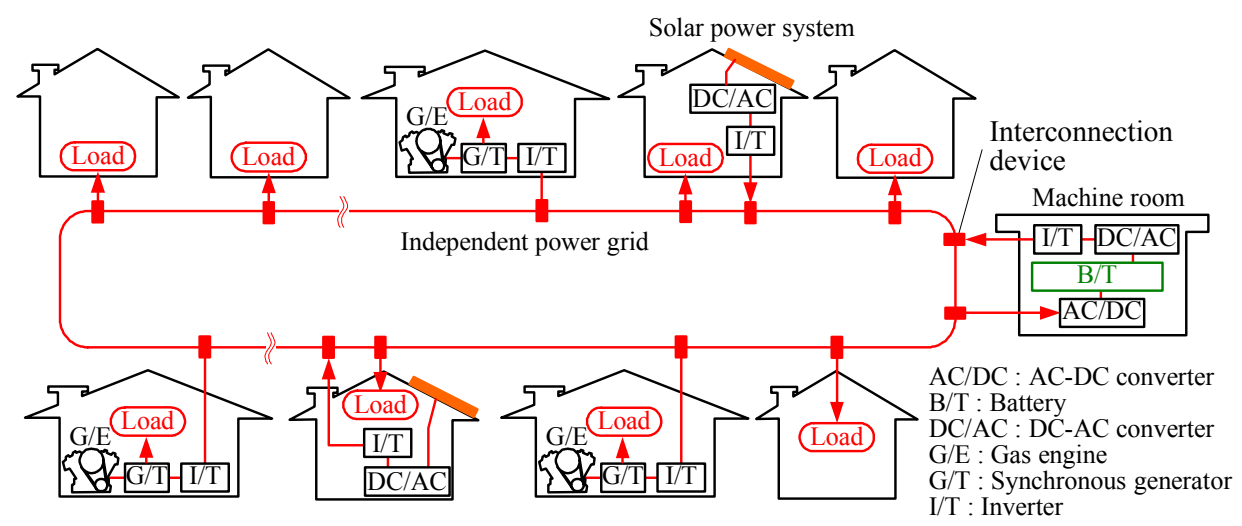

Fig. 1 Independent micro-grid system using gas engines

power storage equipment must be introduced and the dynamic characteristics of the micro-grid must be improved. Due largely to the proliferation of hybrid vehicles and the like, the cost and performance of batteries have recently improved remarkably ${ }^{7)}$. With this in mind, this paper investigates algorithms for the operation planning of a micro-grid that combines conventional type energy equipment, a solar cell and a battery. Since a micro-grid is typically built up of two or more energy systems, we have to solve a nonlinear problem with many variables. In this paper, the operation condition of the generating equipment is expressed with chromosome codes, these are introduced into a GA, and the optimal operation planning is determined.

\section{Independent micro-grid with renewable energy and battery}

\subsection{System configuration}

Figure 1 shows the case of an independent micro-grid that distributes power to a small collection of generating equipment (here we use gas engine generators as an example, but our analysis is not limited to this case). The micro-grid is controlled by a system controller on which we have installed dynamic operation planning software. In this paper, we investigate the power supply-and-demand characteristics of the micro-grid; the examination concerning the supply-and-demand of heat in a similar system will be reported independently.

\subsection{Dynamic operation planning}

(1) Operation planning based on a solar cell output power model

Figure 2 shows the operation method of a micro-grid with a solar cell. Dynamic operation of the micro-grid is planned based on the output power model of a solar cell. In this paper, the solar cell output model is based on PAS predictive data and past average weather data ${ }^{8)-9)}$. The PAS is a production-of-electricity prediction algorithm that was designed specifically for the solar cell in the work ${ }^{5)}$. Figure 2 (a) shows the method of planned dynamic operation based on the output power model of the solar cell described in the introduction. From time $t_{0}$ to time $t_{2}$, there is no output from the solar cell and so in this interval, more electric energy is stored in the battery than is required by the load. In light of this, generating equipment is operated during this period (Generator output (1)). Each piece of generating equipment is operated by the fixed load near the maximum efficiency point. The supply of electric power in the micro-grid is adjusted by controlling the number of pieces of power equipment in operation. When the supply of electric power exceeds the load in the period from $t_{0}$ to $t_{2}$, the amount of surplus charge is moved into the battery. There is an output of the solar cell in the period $t_{3}$ to $t_{6}$. In the period $t_{2}$ to $t_{4}$, the system responds to a load by discharging the battery, and supplying charge from output of the solar cell. Because the output of the solar cell exceeds the load in the period $t_{5}$ to $t_{4}$, surplus power can again be stored in the battery. If the output characteristics of the solar cell and the characteristic of power load are able to be predicted in the early morning, one can minimize the number of equipment hours of operation and the number of charge and discharge cycles on the battery throughout the day. It is known, however, that the load following operation of 
power equipment is disadvantageous when it comes to installed capacity and the hours of operation required of power equipment when compared with the output predictive model of a solar cell ${ }^{5)}$.

(2) Error of the output predictive model of a solar cell, and the influence on an operation plan

Figure 2 (b) shows the method of an operation plan when a difference occurs between the output model of the solar cell shown in Fig. 2 (a), and an actual solar cell output. This system starts out (during $t_{0}$ to $t_{2}$ ) by following the operation plan shown by the command of the controller in Fig. 2 (a). However, as shown in Fig. 2 (b), since there is so little power being produced in the solar cell, the period of $t_{4}$ to $t_{5}$ shown in Fig. 2 (b) requires additional operation of power equipment (Generator output (2)). The system is continuously controlled by the controller to follow the operation shown in Fig. 2 (a). However, there is little storage of electricity by the solar cell in Fig. 2 (b) compared with Fig. 2 (a). For this reason, when discharged from a battery in the period $t_{7}$ to $t_{8}$, the system will change to load following operation (Generator output (3) and (4)). As a result, the number of hours of operation of power equipment increases, and the additional capacity of the battery cannot be sufficiently utilized further.

\subsection{Solar cell system}

The area of power-generation of the solar cell introduced into the micro-grid will be called $S_{s o l}$ and we are assuming a polycrystalline silicon type solar cell. The production of electricity of the solar cell $P_{\text {sol }}$ is calculated using Eq. (1). The power-generation efficiency changes as the temperature of the solar cell $T_{r e f}$ changes (the efficiency falls as the temperature increases). The temperature coefficient in this case is called $R_{T} . T_{\infty}$ is a reference temperature and $\eta_{\text {sol }}$ is the power-generation efficiency of the solar cell at $T_{\infty}$. $H_{D}$ in Eq. (1) expresses the solar radiation intensity for direct delivery (the intensity of radiation which enters into the acceptance surface). Moreover, $H_{S}$ in Eq. (1) expresses the solar radiation intensity of the dispersion component.

$$
P_{\text {sol }}=S_{\text {sol }} \cdot \eta_{\text {sol }} \cdot\left(H_{D}+H_{S}\right) \cdot\left\{1-\left(T_{r e f}-T_{\infty}\right) \cdot\left(R_{T} / 100\right)\right\}
$$

\section{Power balance and objective function}

\subsection{Power balance}

Equation (2) expresses the power balance equation in the proposed micro-grid. The left-hand side of the equation is the power outputted by the composition of equipment that makes up the system, and the right-hand side expresses the power consumed by the micro-grid. $E_{g e n, i, t}, E_{b t, t}$ and $E_{s o l, t}$ of the left-hand side express the output of the generating equipment, battery, and solar cell between time $t$ and $t+1$, respectively. Moreover, $N_{\text {eng }}$ is the number of pieces of generating equipment introduced into the micro-grid. $E_{n e e d, j, t}$ is the power demand at time $t$ of the house $j$. Nhouse is the number of the houses connected to the micro-grid. The last term on the right-hand side of Eq. (2) $\left(\Delta E_{\text {loss,t }}\right)$ expresses the power loss in the system. The charge-and-discharge

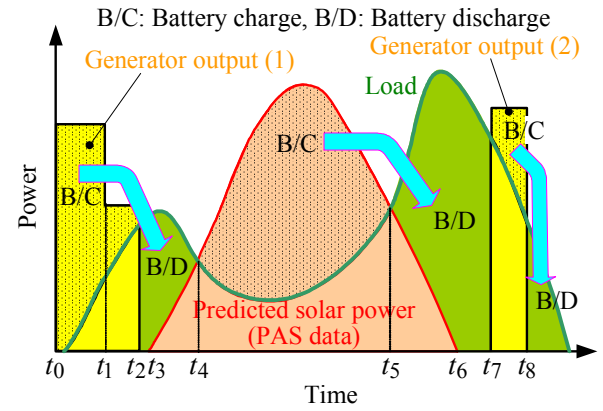

(a) Operation planning based on the PAS predictive data

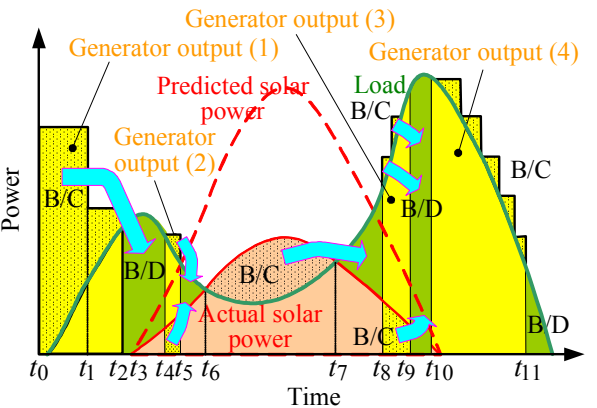

(b) Operation planning when taking into consideration the error between PAS predictive data and actual solar power

Fig. 2 Operation planning of a micro grid with a solar power 
efficiency of a battery, power transmission loss, etc., are included in this term. In the analysis of this paper, the charge-and-discharge efficiency of a battery is the only effect included in this term. The sampling interval for each piece of equipment, such as the generating equipment, a solar cell, or a battery, is set to be one hour in this paper.

$$
\sum_{i=1}^{N_{\text {eng }}} E_{\text {gen }, i, t}+E_{b t, t}+E_{\text {sol }, t}=\sum_{j=1}^{N_{\text {house }}} E_{\text {need }, j, t}+\Delta E_{\text {loss }, t}
$$

\subsection{Objective function}

The number of hours of operation of the generating equipment EOTt between time $t$ to $t+1$ is obtained by calculating Eq. (3). However, $n_{g e n, i, t}$ expresses the operational status of the generating equipment $i$ in the time between $t$ and $t+1$ (" 1 " indicates operation, "0" indicates idle). $N_{\text {gen }}$ is the number of pieces of generating equipment installed in the micro-grid. The total number of hours of operation for all pieces of generating equipment that were in operation during the periods $t=1,2, \ldots . P_{s y s}$ is calculated by Eq. (4). The optimization of the dynamic operation plan of the micro-grid is examined using a genetic algorithm (GA). In the GA, the objective function shown by Eq (4) is defined as an adaptive value. The solution that more closely satisfies Eq. (4) is described as having a "large adaptive value."

$$
\mathrm{EOT}_{t}=\sum_{i=1}^{\text {Ngen }} n_{g e n, i, t}
$$

Engine operation time $=\operatorname{minimize}\left(\sum_{t=1}^{P_{s y s}} E O T_{t}\right)$

\section{Analysis method}

4.1 Production-of-electricity prediction algorithm of solar cell (PAS) ${ }^{5)}$

A layered neural network (NN) is introduced and the production of electricity of a solar cell is predicted according to the following procedures.

(1) Input-and-output data of NN used by PAS

Figure 3 expresses the input-and-output data of the NN used for PAS. $d w$ expresses the present date and $t$ expresses the present time. In the learning and analysis process of the $\mathrm{NN}$, the average amount of solar radiation and average outdoor air temperature are inputted as data for each time of the present date. The input data described in the introduction is fed into Eq. (1), and the production of electricity $P_{s o l}$ of the solar cell is obtained. This $P_{\text {sol }}$ used as the teaching data in the learning process of the NN.

(2) Input data

The data inputted into the NN the learning process includes the average amount of solar radiation and average outdoor air temperature for each time of the day (from time 0 to time $t$ ). On the other hand, the average amount of solar radiation and average outdoor air temperature from $t$ to 24 of a given day give the values measured on the same time and the same day of previous years, as obtained from "the standard weather and the solar

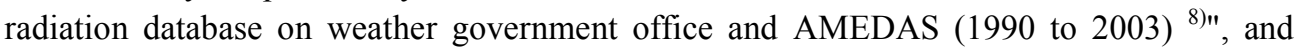
"NEDO technical information data base (METPV-3) 9)". The data inputted into the NN during the analysis process are the same as those inputted during the learning process from 0 to $t$. The average amount of solar radiation and average outdoor air temperature from $t$ to 24 of a given day give the data measured at same time the previous day.

(3) Structure of the NN, and output data

Figure 4 shows the structure of the NN introduced into PAS. Figure 4 (a) shows the learning process and Fig. 4 (b) shows the analysis process. The NN used in this proposal has three layers. In the learning process, the input data ( $x_{1}$ to $\left.x_{48}\right)$ (described in Section (2)) 


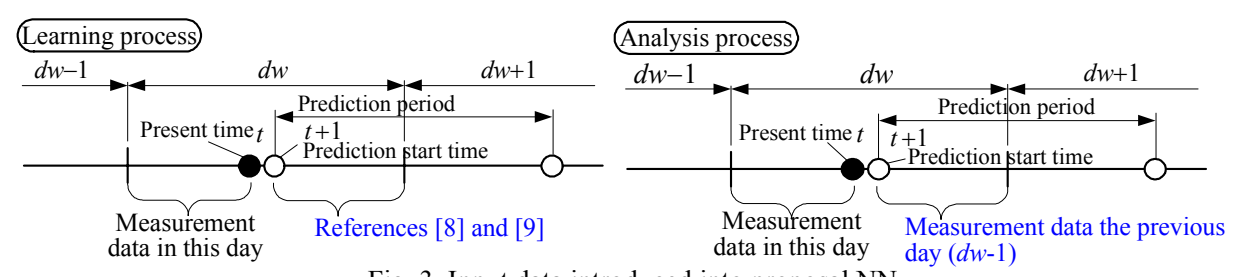

Fig. 3 Input data introduced into proposal NN

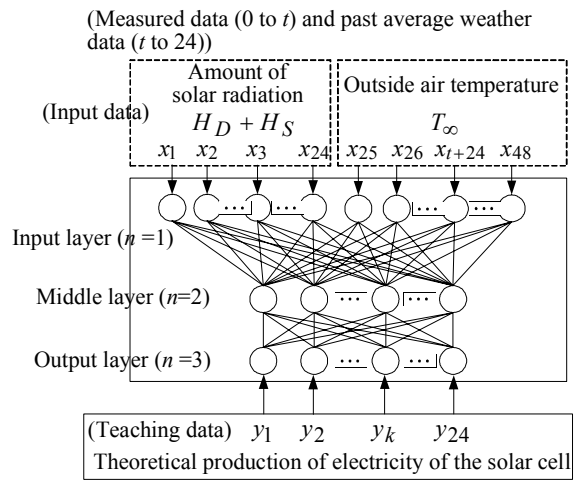

(a) Learning process

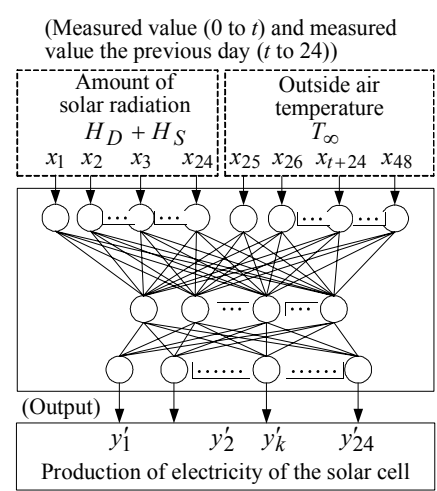

(b) Analysis process

Fig. 4 Layered neural network of the proposed system

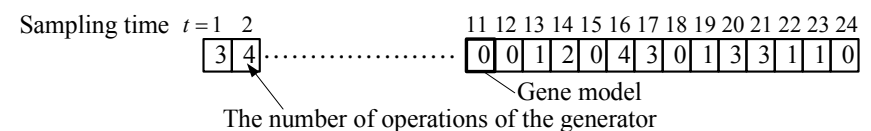

Fig. 5 Chromosome code used for genetic algorithm

are fed into the first layer (the power-input layer) and the teaching data ( $y_{1}$ to $\left.y_{24}\right)$ are fed into the third layer (the output layer). The weight of each network connection between neurons is decided using back propagation ${ }^{10)}$ so that the relationship between each input data point and each teaching data point may be realized. Input data can be given to a learned $\mathrm{NN}$ (in the analysis process), and the solar cell output power ( $y_{1}^{\prime}$ to $y_{24}^{\prime}$ ) in each time of $d w$ can be obtained from the output layer.

4.2 Optimization of dynamic operation using a genetic algorithm (GA)

\subsubsection{Chromosome Model}

Dynamic operation planning of the micro-grid is optimized using a GA based on the solar cell output power model (the PAS predictive data and the past average weather data). Figure 5 expresses the chromosome code used by the proposed GA. One chromosome (individual) consists of 24 genes. Each gene shows the operational status of the various pieces of generating equipment that are on the grid during each time step in the date $d w$. With the number of the pieces of generating equipment introduced into the grid being called $N_{\text {gen }}$, the value of each gene is an integer between 0 and $N_{\text {gen }}$. The genes of the initial generation's chromosome are decided using random numbers.

\subsubsection{Multiplication and Selection}

By decoding the genes in a chromosome, the operation condition $n_{g e n, i, t}$ of the generating equipment in Time $t$ can be obtained. By giving $n_{\text {gen }, i, t}$ to Eq. (3), EOTt is calculable. Furthermore, the value of the objective function of each chromosome is calculated from Eq. (4). The software implementation is such that the number of individuals of high adaptive value (gotten by evaluating of the objective function) may be multiplied at a fixed rate.

\subsubsection{Crossover}

Change of generation is repeated, adding the genetic manipulation of crossover to chromosomes to maintain diversity (an evaluative process). In the chromosome code, when arriving at the last generation, a fitness value decides the individual most suited to be the optimal operating method. In the crossover calculation, two parent chromosomes are selected by the crossover probability $P_{\text {cros }}$ given beforehand, and the crossover position common to both of parent chromosomes is decided at random. The genes of both of the 


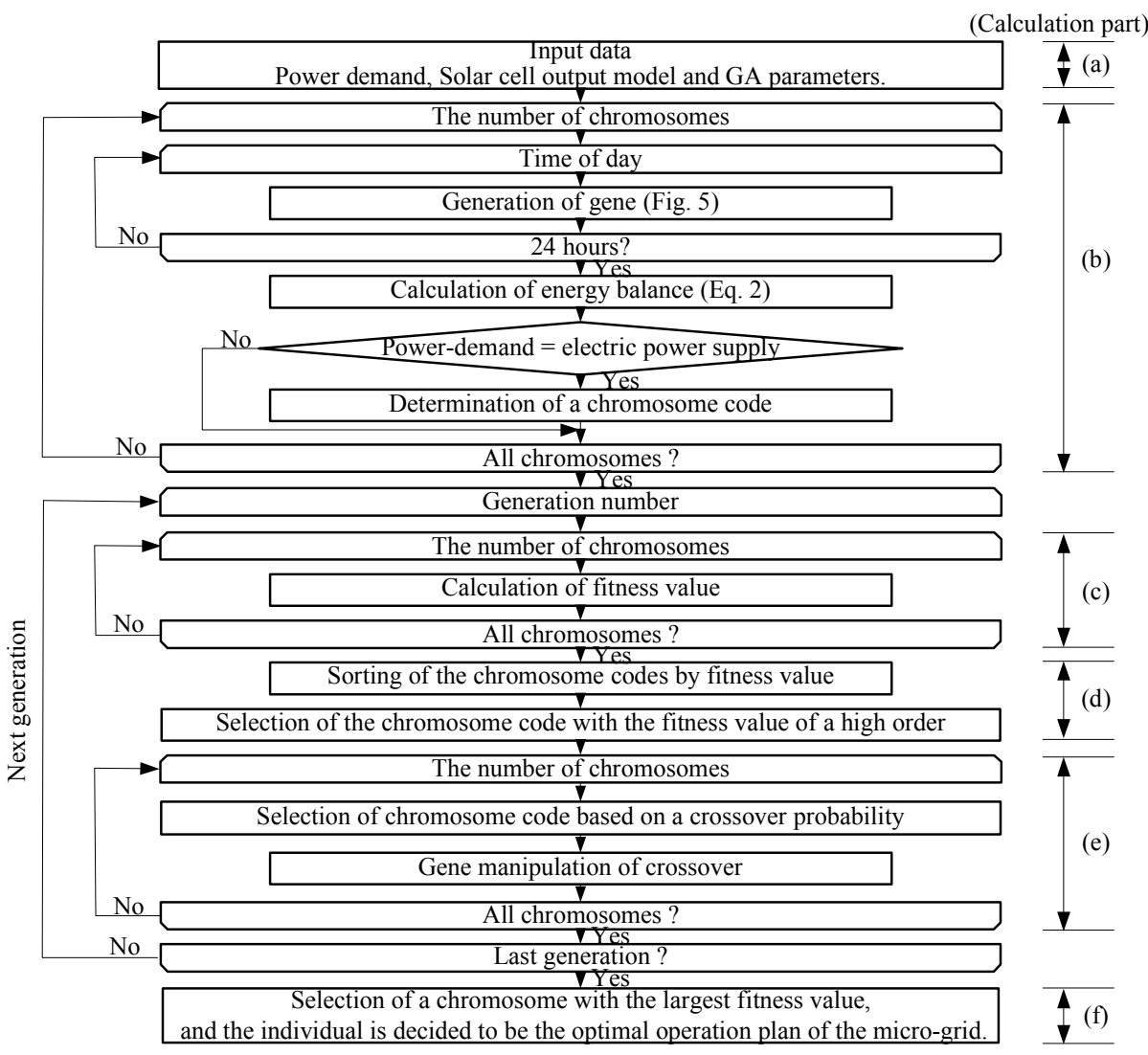

Fig. 6 Calculation flow of the optimal operation plan using GA

parent chromosomes are rearranged bordering on the crossover position, and the chromosome with a new gene is generated.

\subsection{Analysis flow of operation planning}

Analysis flow of the operation planned optimization of the micro-grid by the proposal GA is shown in Fig. 6. In the Calculation part (a) of the figure, an electricity demand pattern, a solar cell output power model (PAS or output power pattern of the solar cell based on the past average weather data), and the parameter of GA are inputted. The initial generation's chromosome group is generated in Calculation part (b). In Calculation part (c), the fitness value of all the chromosomes is calculated and the order of chromosomes is decided at Calculation part (d) according to the magnitude of their fitness values. Chromosomes of large fitness value are made to increase in number at a fixed rate, while chromosomes of small fitness value are deleted. In Calculation part (e), a parent chromosome is chosen at random under crossover probability, a crossover position is decided at random, and genes are exchanged. In Calculation part (c), (d), (e), the calculation of the fitness value, the operation of arranging chromosomes in order of fitness value, and exchange of the gene by crossover is done. These operations are calculated and repeated until we reach the final generation number. In the last generation's chromosomes, an individual with the highest fitness value is decided to be the optimal solution. By decoding the chromosome code of the optimal solution, operation of the generating equipment in each time step of date $d w$ can be planned.

\section{Case analysis}

\subsection{Analysis System}

In this case analysis, we assume the introduction of the micro-grid into nine houses in Sapporo. In this Section, the difference of the operation plan when introducing the proposal method or the past average weather data is investigated. The generating equipment introduced into the micro-grid shall be structured in five sets, and the power output of each 


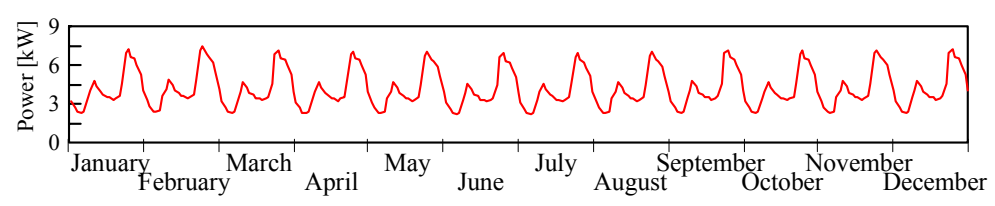

Fig. 7 Power demand of the nine-houses micro-grid in Sapporo
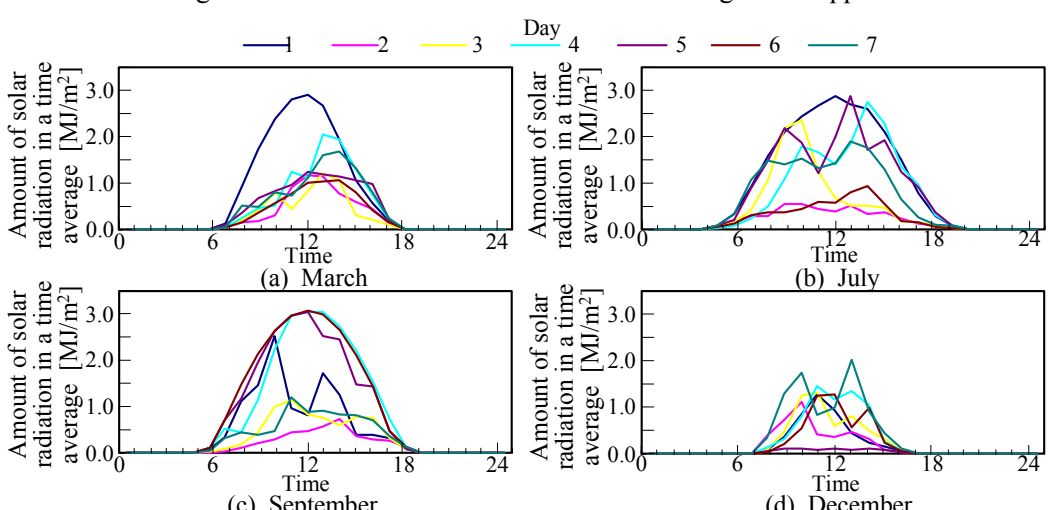

Fig. 8 The slope-face amount of solar radiation in Sapporo in 1990 to 2004 (30 degrees of the angle, for south)

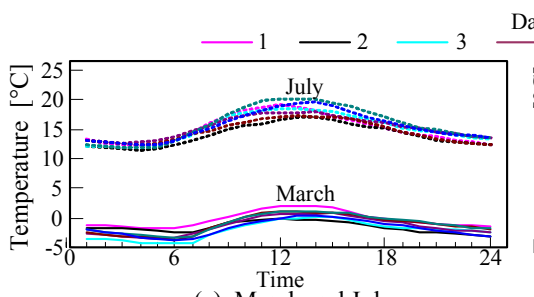

(a) March and July (d) December
ro in 1990 to 2004

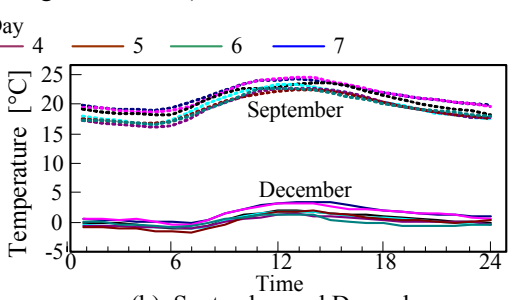

(b) September and December

Fig. 9 Outside temperature data in Sapporo in 1990 to 2004

piece of generating equipment shall be $1 \mathrm{~kW}$. As described in Section 5.2, the power load introduced into this case analysis has a maximum of about $7.5 \mathrm{~kW}$ (Fig. 7).

The solar cell is a flat plate type of polycrystalline silicon, and installs the cell of area 150 $\mathrm{m}^{2}$ facing south by sloping 30 degrees. Since the area of the solar cell installed in an average house is 20 to $40 \mathrm{~m}^{2}$, the capacity of the solar cell linked to the proposal micro-grid is equivalent to 4 to 7 houses. The battery introduced into the system is a nickel hydrogen type. The performance and specification were decided from the reference ${ }^{10}$. The analysis conditions of the solar cell, battery, each converter used in the simulation, and an inverter are shown in Table 1. The loss of charge efficiency, discharge efficiency, and natural discharge are all included in the table.

\subsection{Analysis Conditions}

(1) Power load

The time average of the electricity demand pattern of nine houses in the representation day of the every month of Sapporo is shown in Fig. $7^{11}$. Air conditioning load is not included in the electricity demand pattern of Fig. 7 and it is assumed that there are four residents per house on the average. Space heating load is supplied with engine exhaust heat, and cooling load is unnecessary. Although the actual electricity demand pattern changes sharply on short time scales, a gradually varying time-averaged value is used throughout this paper.

(2) Amount of solar radiation and outdoor air temperature

In this case analysis we will investigate about six days, from the 2nd day to 7 th day in each of March, June, September, and December (the 1st day of every month is used to check the prediction of the PAS analysis). Figures 8 and 9 show the measured amount of solar radiation and outside temperature for seven days in every month from 1990 to 2004 in Sapporo ${ }^{8-99}$. The daily fluctuations in the amount of solar radiation are typically large when compared to the daily fluctuations of the outdoor air temperature in every month.

(3) GA parameters

In this case analysis, the number of an initial generation's chromosome codes is 8000 . In 
Table 1 Specifications of equipments

\begin{tabular}{|lr|}
\hline - Solar cell type & Multicrystalline silicon \\
- Generation efficiency & $14 \%$ \\
of the solar cell & \\
- Temperature coefficient & $0.4 \% / \mathrm{K}$ \\
of the solar cell & \\
- Battery type & Nickel-Hydrogen \\
- Battery efficiency & $90 \%$ \\
- Efficiency of AC-DC converter & $95 \%$ \\
- Efficiency of DC-AC converter & $95 \%$ \\
- Efficiency of DC-DC converter & $95 \%$ \\
\hline
\end{tabular}

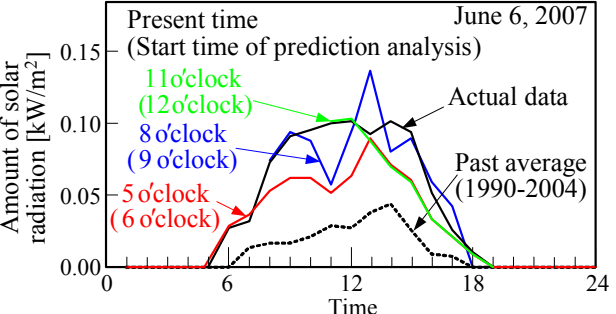

Fig. 10 Solar radiation in 30-degree slope of the south sense using PAS, and actual solar radiation. Prediction start time of PAS, and the characteristic of prediction solar radiation.
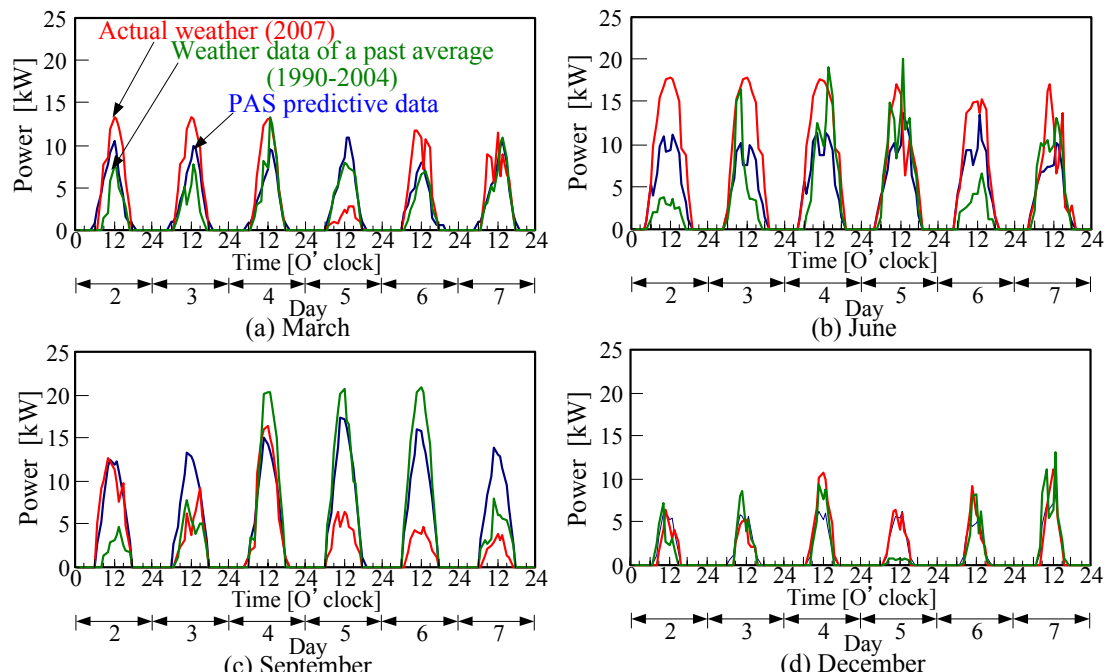

Fig. 11 PAS prediction results and the actual value of the solar cell output in Sapporo

the genetic manipulation of multiplication and selection, the 20 chromosome codes (that is, $5 \%$ of the population) with the largest fitness values are made to increase their number in the next generation. The crossover probability is set to 0.2 . The generation number was set to 10 because of the large total number of chromosome codes. These parameters of GA were arrived at by a trial-and-error method, and the final values decided using analysis accuracy as a reference.

\section{Analysis results}

\subsection{Prediction of solar cell output power via PAS}

(1) Relationship between prediction start time and analysis accuracy

Figure 10 shows the predicted amount of solar radiation on June 6, 2007 in a south-facing set of 30 distinct angular orientations, calculated using PAS. In Fig. 10, it will be the present time in 5,8 , and 11 , and will be each prediction start time in 6,9 , and 12 . Actual data refers to the weather data measured on June 6,2007 . We see that the difference between past average weather data ${ }^{8-9)}$ and actual data is larger than the difference between the actual data and the predicted results. Moreover, the data that came from using a start time of 9 or 12 are closer to the actual data than that which came from using a start time of 6 . In the result of Fig. 10, if there is a large power input when using the weather data measured at that day (accordingly, the prediction start time is late), the analysis accuracy will have improved. In the analysis using PAS or later, prediction start time is 6 o'clock.

(2) Prediction result in every month

The result based on the prediction result of solar cell output power, actual value, and past average weather data ${ }^{8)-9)}$ of having used PAS in representation days every month is shown in Fig. 11. One can see from Fig. 11 that in many cases one gets closer to the actual value by using the PAS prediction than one would get by using past average weather data.

6.2 Prediction error of PAS, and operation method of generating equipment

6.2.1 Operation planning of generating equipment

Figure 12 shows the result of the dynamic operations analysis on March 2, 2007. In order 


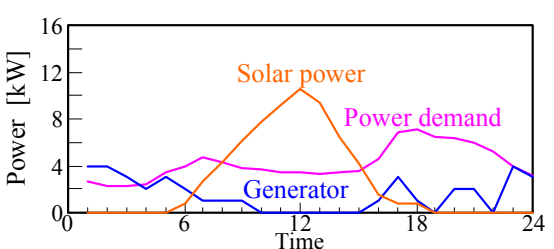

(a) Operation plan based on the PAS predictive data

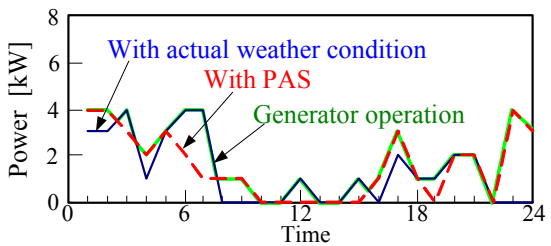

(c) Dynamic operation planning of the generato using the PAS predictive data and the actual weather

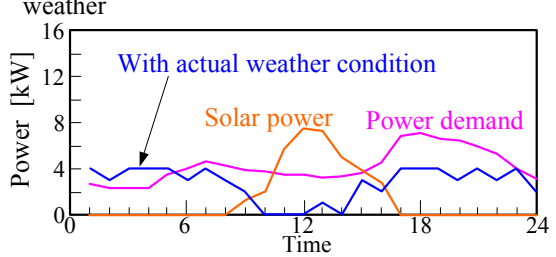

(e) Operation plan based on the weather data of a past average

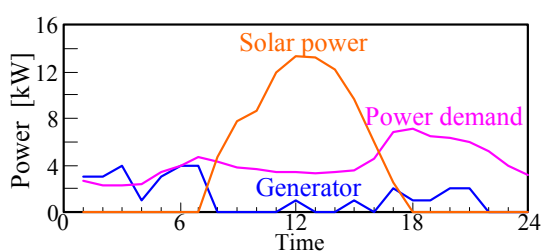

(b) Operation plan using actual weather data

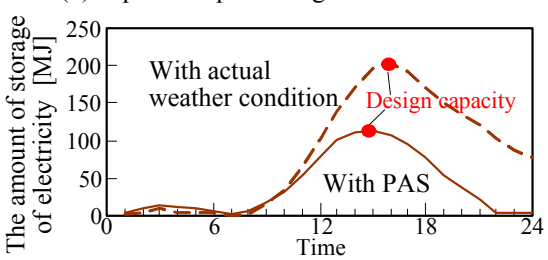

(d) Operation plan of the amount of storage of electricity in the battery

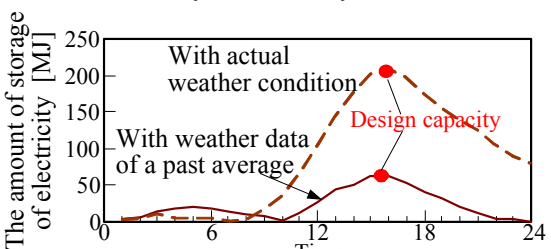

(f) Operation plan of the amount of storage of electricity in the battery based on the weather data of a past average

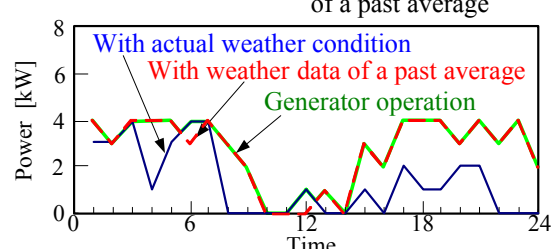

(g) Dynamic operation planning of the generator using the weather data of a past average and the actual weather

Fig. 12 Result of the dynamic operation plan of the micro-grid system (March 2, 2007)

to investigate the influence of operation planning on battery efficiency, we here set the battery efficiency to $100 \%$. The battery efficiency in Table 1 means charge-and-discharge efficiency. In analyses other than Fig. 12, the battery efficiency shown in Table 1 is used. Accordingly, the loss based on battery efficiency is not taken into consideration in Fig. 12. Figure 12 shows the calculation result except charge-and-discharge loss of the battery, in order to clarify the power balance about consumption, the solar cell, and the generator equipment. Fig. 12 (a) shows the result of operation planning of generating equipment using the solar cell output power prediction generated by PAS. On the other hand, Fig. 12 (b) shows the result of operation planning of generating equipment using the actual amount of solar radiation and outdoor air temperature. If the PAS generated solar cell output power is the same as that coming from actual data then the optimal operation planning method is shown in Fig. 12 (a). However, one must also note that errors are introduced in the PAS predictions and actual operation may more closely resemble the result of Fig. 12 (c). Accordingly, the output power of the generating equipment as controlled by PAS and that controlled by actual weather conditions are compared, and the system is operated in such a way as to accommodate the worst conditions (this is a typical way of doing things in similar systems).

\subsubsection{Operation planning of battery}

Figure 12 (d) shows the results that come from the operation planning of a battery when using either PAS predictions or actual data to calculate the solar cell output power. One sees that the operating characteristics of the battery greatly influence the method and duration of operation of the generating equipment. Looking at Fig. 12 (d), one sees that, in at least two different places, there is a significant difference in the battery capacity calculated in the two different schemes.

6.2.3 Output characteristics of the solar cell, and operation planning

Figure 12 (e) shows the operation planning of the generating equipment when using 

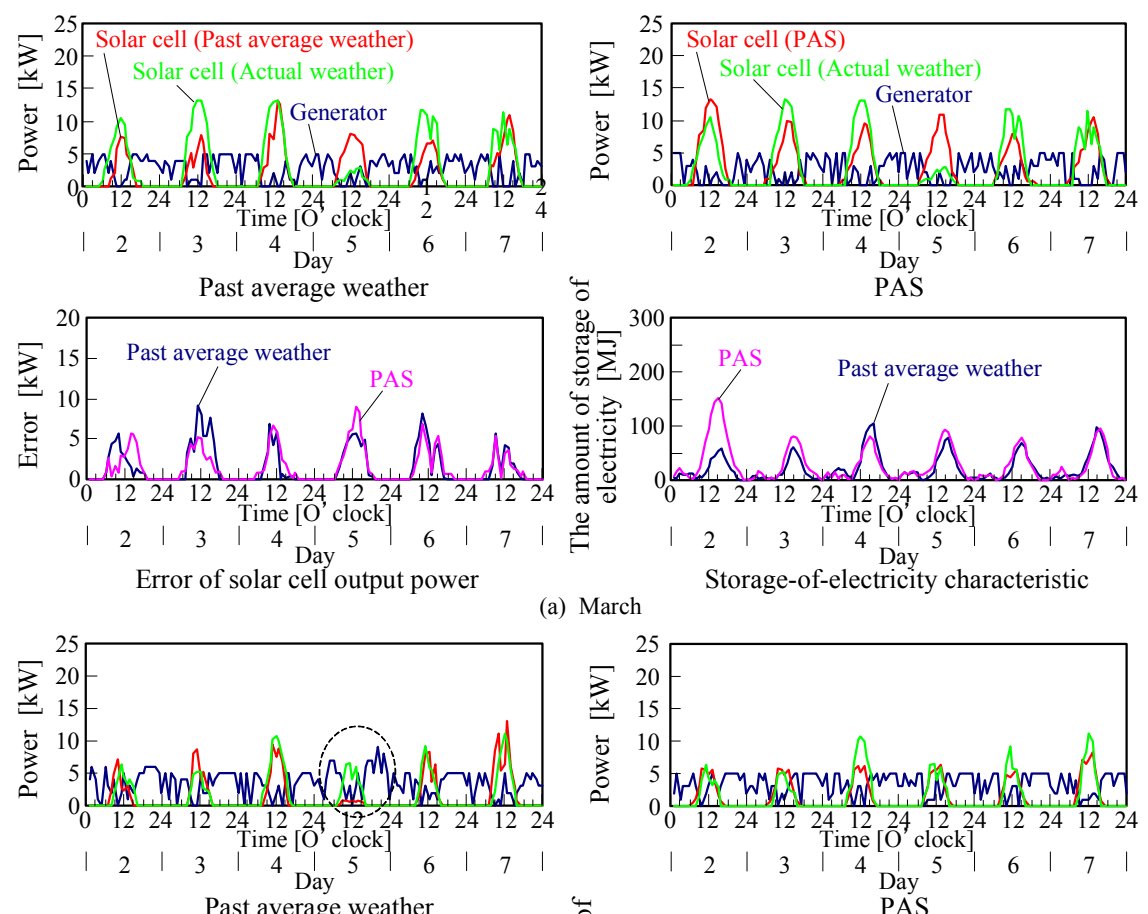

a) March

Storage-of-electricity characteristic
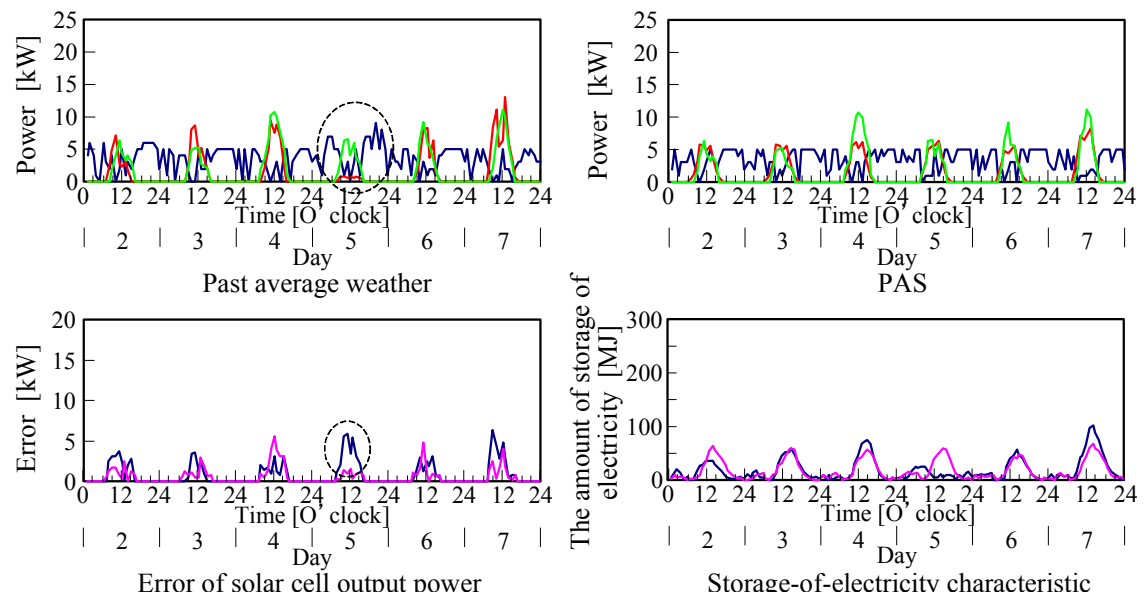

(b) December

Storage-of-electricity characteristic

Fig. 13 Dynamic operation planning based on the past average weather data and the PAS predicted data. In these analyses, the influence on operation planning by the error of "the past average weather data" and "the PAS predictive data" to actual weather is not taking into consideration.

either past average weather data or actual weather data. Figure 12 (f) shows the resultant battery capacity planning under the same conditions. Fig. 12 (g) shows the result of operation planning of generating equipment. When Fig. 12 (c) is compared with Fig. 12 (g), one sees that distinct methods of planning for generating equipment begin to deliver noticeably divergent results after about time 15 . This happens as the output power models of the solar cell introduced into operation planning begin to produce diverging results. Accordingly, the accuracy of the output power prediction generated by PAS has a large influence on operation planning of the generating equipment the capacity planning of the battery.

6.3 Result of dynamic operation planning

6.3.1 Operation planning of micro-grid based on the PAS and the past average weather data

Figure 13, shows the result of dynamic operation planning of the system (including generating equipment and batteries) when using either past average weather data or PAS prediction data as the solar cell output power model. The error in the solar cell output power in Fig. 13 is the difference between the output determined by a given simulation and that determined from actual weather conditions. In operation of an actual micro-grid, a difference is in the solar cell output power model described in the introduction, and the solar cell output power operating under actual weather conditions. As Section 6.2.1 described, in dynamic operations planning using the solar cell output power model, additional operation of generating equipment is expected in an unfavorable condition. For example we see, in Fig. 13 (b), that the partial output power of the generating equipment on December 5 (calculated in the past average weather data scheme) exceeds $5 \mathrm{~kW}$, the maximum power of 


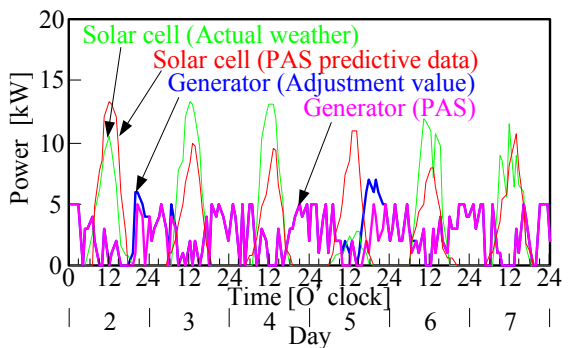

(a) March

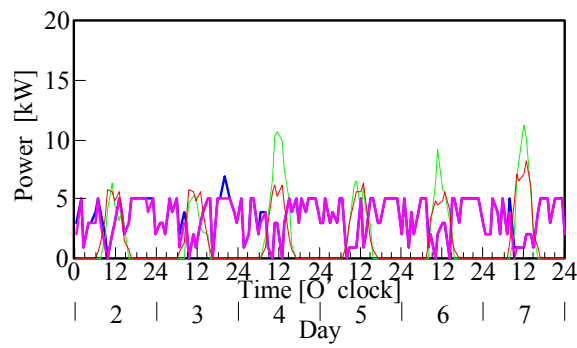

(b) December

Fig. 14 Results of the dynamic operation planning of the power generator with PAS prediction. The error of the PAS predictive data to the actual weather is taking into consideration.

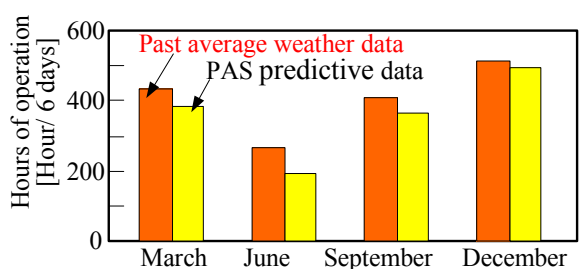

Fig. 15 Result of the generator hours of operation analyzed under the past average weather data and the PAS predictive data. Consideration with error to the actual value is added.

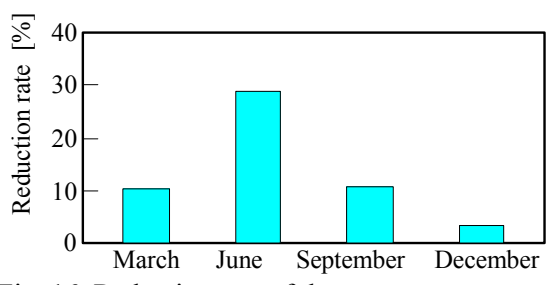

Fig. 16 Reduction rate of the power generator operation hours in the case of introducing the PAS predictive data to the past average weather data. Consideration with error to the actual value is added.

the proposed system. We can make sense of this as the smallest amount of solar radiation comes in the month of December. Because of this, the solar cell output power grows small and the discrepancy with past average weather data grows large. Thus, if the error between the model output power and the actual output power is large, the working time of the generating equipment is expected to increase. In comparison, for operations planning using the PAS predictive data (see Fig. 13), the generating equipment is never asked to exceed 5 $\mathrm{kW}$ in the entire month of December. We see here that operations planning by PAS is at an advantage over planning with past average weather data.

6.3.2 Actual system operation using the PAS

If a difference occurs in the solar cell output power based on the PAS predictive value and actual weather conditions, the operation of generating equipment will follow the method described below. Power balance (Eq. (2)) of the micro-grid in the sampling time $t$ is calculated, and when electricity demand exceeds supply, the generating equipment starts operation immediately. The amount of pieces of generating equipment sent into operation at this time is decided to be the minimum number plausible to avoid the case where the power supply exceeds the demand. However, it is not this limitation when large electricity demand is predicted at later time from the analysis of dynamic operation planning in that case. Accordingly, when demand is expected to exceed the maximum power supply $(5 \mathrm{~kW})$, we can increase the number of pieces of equipment in operation beforehand. Figure 14 shows the operation of the generating equipments and battery as a result of adding the modification (the operation method described in the above) concerning a prediction error to operation planning using PAS shown in Fig. 13. Figure 14 shows operation planning of the generating equipment using PAS predictive data, as well as operation planning with adjustment of PAS prediction error (here called "Adjustment value"). In actual operation of a micro-grid, operation planning with PAS and with "operation planning of the generating equipment by PAS predictive data" and "operation plans by adjustment value" in each sampling time are compared, while a large power output is being performed.

\subsubsection{Hours of operation of generating equipment}

Figure 15 shows the result of operating the generating equipment in the micro-grid according to the method described in Section 6.3.2. When the hours of operation using past average weather data and PAS prediction data are compared, there is few hours worked method when introducing PAS to all the month. The advantageous operation method can be obtain from this rather than the operation planning method using the past average weather deta. Moreover, Fig. 16 shows the reduction in the total equipment working hours due to using the PAS prediction data rather than using the past average weather data. By 
introducing PAS into dynamic operation planning of the micro-grid, the working time of generating equipment is reduced, as compared to using past average weather data, from $3 \%$ to $30 \%$.

\section{Conclusions}

The operations planning of the micro-grid was investigated here using a proposed algorithm. Operation of the proposed micro-grid was analyzed using actual weather data (amount of solar radiation and outside temperature) collected from the 1 st to the 7 th in the months March, June, September, and December of 2007 in Sapporo. However, it is thought that the following results change by the introductory region of the micro grid. If the analysis method described in this paper is introduced into various places, the micro-grid system in consideration of regionality will be constructed. The following conclusions have been obtained:

(1) If the PAS predictive value is introduced as the predictive value of solar cell output power compared with the past average weather data, the working time of the generating equipment can be reduced from $30 \%$ to $3 \%$. However, working time of generating equipment is under the influenced of battery capacity.

(2) However, there is the possibility that in seasons with only a small amount of solar radiation, if the prediction error of PAS is large, the original operation planning will change greatly. In this case, a storage capacity over and above the capability of the generating equipment introduced into the micro-grid is predicted.

When introducing the proposal analysis method into areas other than Sapporo, it is necessary to investigate the relation between following important factors and system operation plan.

a. Influence of space cooling load and space heating load.

b. Influence of a rainy season with a small amount of solar radiation.

\section{References}

(1) Abu-Sharkh, et al., Can Microgrids Make a Major Contribution to UK Energy Supply? Renewable and Sustainable Energy Reviews, Vol. 10, No. 2(2006), pp.78-127.

(2) M. Muselli, G. Notton and A. Louche, Design of Hybrid-Photovoltaic Power Generator, With Optimization of Energy Management, Solar Energy, Vol. 65, No. 3(1999), pp. 143-157.

(3) Ismail Y, Kemmoku Y, Takikawa H and Sakakibara T, An Operating Method for Fuel Savings in a Stand-Alone Wind/Diesel/Battery System, Journal of Japan Solar Energy Society, Vol. 28, No. 2(2002), pp.31-38.

(4) Shin'ya OBARA, Operating Schedule of a Combined Energy Network System with Fuel Cell, International Journal of Energy Research, Vol. 30, No. 13(2006), pp.1055-1073.

(5) Shin'ya OBARA and Itaru TANNO, Fuel Reduction Effect of the Solar Cell and Diesel Engine Hybrid System with a Prediction Algorithm of Solar Power Generation, Journal of Power and Energy Systems, Vol. 2, No. 4(2008), pp.1166-1177.

(6) Shin'ya OBARA, Dynamic Operation Plan of a Combined Fuel Cell Cogeneration, Solar Module, and Geo-Thermal Heat Pump System Using Genetic Algorithm, International Journal of Energy Research, Vol. 31, No. 13(2007), pp.1275-1291.

(7) K. Jorgensen, Technologies for electric, hybrid and hydrogen vehicles: Electricity from renewable energy sources in transport, Utilities Policy, Vol. 16, No. 2(2008), pp. 72-79.

(8) Homepage of Japan Meteorological Agency, 2007, http://www.data.jma.go.jp/obd/stats/etrn/index.php

(9) NEDO Technical information data base, Standard meteorology and solar radiation data (METPV-3), http://www.nedo.go.jp/database/index.html

(10) Shin'ya OBARA and Itaru TANNO, Operation Prediction of a Bioethanol Solar Reforming System Using a Neural Network, Journal of Thermal Science and Technology, Vol. 2, No. 2(2007), pp.256-267.

(11) Narita, K., The Research on Unused Energy of the Cold Region City and Utilization for the District Heat and Cooling, Ph.D. thesis, (1996), Hokkaido University. 